\title{
Echocardiographic findings in a patient with Takotsubo syndrome: importance of measurements beyond the ejection fraction
}

\begin{abstract}
Takotsubo syndrome refers to an acute heart failure syndrome that mainly affects postmenopausal women, which is characterized by abnormalities in ventricular segmental contractility accompanied by a reduction in the ejection fraction, which is completely reversible. Although the first case of this disease was described 20 years ago, at present we only have information from observational and descriptive studies, with which in 2016 the European Society of Cardiology publishes a state of the art of this medical condition. We present the case of a 65 -year-old woman who consults for chest pain with typical features with elevation of cardiac biomarkers and severe hypokinesia in all apical segments in the echocardiogram, which resolved spontaneously as evidenced in the follow-up images. We present not only the conventional measures but also the global longitudinal strain that shows a characteristic pattern in this type of patients.
\end{abstract}

Keywords: Takotsubo Cardiomyopathy, Hypokinesia, Echocardiography, Magnetic Resonance Imaging
Volume II Issue 2 - 2018

\author{
Luis Silva,' Nelson Pérez, ${ }^{2}$ Verónica Giraldo, ${ }^{3}$ \\ Andrés Duarte, ${ }^{3}$ Gustavo Palomino, ${ }^{4}$ Oscar \\ Pacheco ${ }^{4}$ \\ IInternist, Fellow Cardiology, Military University New Granada - \\ Central Military Hospital, Colombia \\ ${ }^{2}$ Internist, Cardiologist, Intensivist, Central Military Hospital, \\ Colombia \\ ${ }^{3}$ Resident First Year, Specialization in Internal Medicine, Military \\ University New Granada - Central Military Hospital, Colombia \\ ${ }^{4}$ Internist, Cardiologist, Central Military Hospital, Colombia
}

Correspondence: Luis Silva, Internist, Fellow Cardiology, Military University New Granada - Central Military Hospital, Colombia, Email lesilvadg@gmail.com

Received: February 28, 2018 | Published: March 16, 2018

\section{Introduction}

Takotsubo syndrome is a syndrome of heart failure, usually acute, reversible, initially described in the 1990s by Sato and colleagues for the way the left ventricle acquired ventriculography during cardiac catheterization. ${ }^{1,2}$ At that time, the surge due to invasive stratification and primary angioplasty allowed access to a catheterization room for patients with acute coronary syndrome, allowing the presence of a heart "abalonado" in the ventriculogram with contractility disorders but with epicardial arteries without angiographically documented lesions despite the electrocardiographic findings that simulated an acute myocardial infarction with ST segment elevation in the anteroseptal face..$^{2-5}$

Althoughmorethan 2 decadesafteritsinitialdescription, thecurrently available evidence for this type of cardiac involvement comes from case reports, case series, observational cohorts, and reports of national Takotsubo syndrome registries. The European Society of Cardiology (ESC) in the year 2016 publishes a state of the art of the disease where it proposes an algorithm for diagnosis and treatment of this disease. ${ }^{6}$ Within the diagnostic algorithm proposed by the ESC, coronary angiography with left ventriculography should be performed urgently to rule out coronary disease. If there is no compromise in angiography, it should be confirmed that it complies with the diagnostic criteria and indicates an early imaging is an echocardiogram or cardiac magnetic resonance with late enhancement. ${ }^{6,7}$

Through transthoracic echocardiography as the initial image, left ventricular morphology and function, anatomical variants, possible complications (left ventricular outflow tract obstruction, mitral insufficiency, right ventricular involvement, thrombus formation and rupture cardiac) and follow up during recovery. ${ }^{6,8-10}$ The following is a case of Takotsubo syndrome in post- menopausal syndrome in which the specific echocardiographic findings are described in this case and the importance of performing additional measurements to conventional ones such as the overall longitudinal strain.

\section{Presentation of the case}

A 65-year-old female admitted to the Central Military Hospital (HMC) for a 24-hour period of progression consisting of slow onset thoracic pain with maximum intensity at the hour after emotional, oppressive, retrosternal, intense, irradiated stress upper limb and neck, associated with dyspnoea and dysautonomic symptoms. Consultation at a health center where studies are carried out with non-diagnostic electrocardiogram (Figure 1), report of a cardiac biomarker that is positively elevated, 5 times the cut-off point and echocardiogram with segmental disorders of contractility. Treatment is started for Acute Coronary Syndrome without Elevation of the ST Segment and refer to our institution.

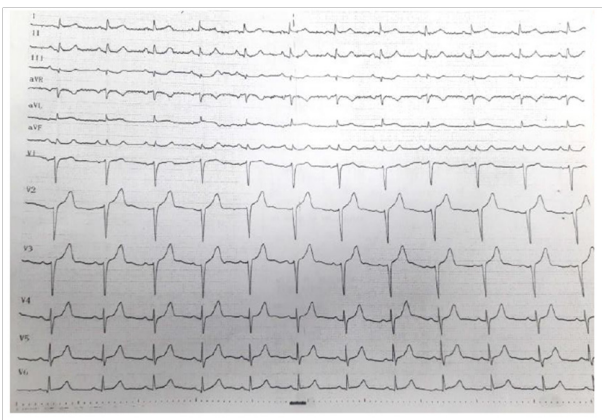

Figure I Initial electrocardiogram. 
On admission to HMC, the patient referred to persistence of pain of the described characteristics, which is present with an intensity of $4 / 10$ and is not associated at that moment with the dysautonomic symptoms presented at the beginning of the picture. Within the systems review, it presents a NYHA functional class I-II/IV, without orthopnea and chest pain prior to this event. As an important antecedent, the patient is diagnosed with Sjögren's Syndrome on treatment with pilocarpine, chronic costochondritis and recurrent urinary tract infections.

Among the studies performed on the patient, a positive troponin report was obtained in the initial care center with a delta less than $20 \%$. Hemoglobin, coagulation times, electrolytes, renal function and glycemia were performed in the HMC, which were within normal limits. We documented by transthoracic echocardiogram performed in our institution a mildly decreased left ventricular systolic function (43\%) due to hypokinesia (Figure 2). In all apical segments, both apical and lateral apical segments were present in all apical segments, as well as an overall longitudinal strain of $-16 \%$, being $-7 \%$ and $-11 \%$ in all apical segments.

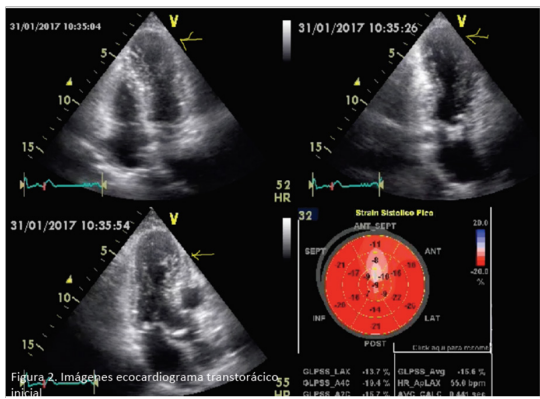

Figure 2 Initial transthoracic echocardiography images.

With the findings of the echocardiogram, the patient is taken to a coronary arteriography where no significant lesions are documented and in the ventriculogram an apical abalonamiento with significant involvement of the ejection fraction is observed. Takotsubo syndrome was considered compatible, so the pharmacological management of the patient was adjusted and a new assessment was scheduled with electrocardiogram (Figure 3) and transthoracic echocardiogram at the month of the event.

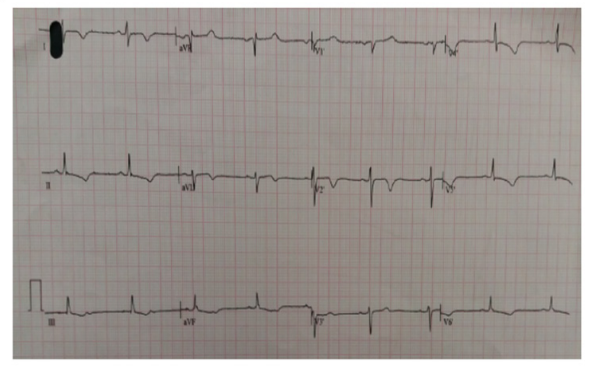

Figure 3 Control electrocardiogram at the month of the event.

On the new echocardiogram, a $60 \%$ left ventricular ejection fraction without segmental contractility disorders was documented with an overall longitudinal strain of $-21 \%$, which is within normal limits (Figure 4). The patient is currently asymptomatic.

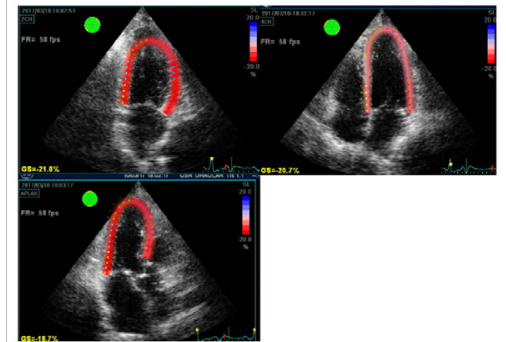

Figure 4 Transthoracic echocardiogram monitoring at the month of the event.

\section{Help}

In the last 25 years, Takotsubo syndrome (STT) has been recognized as a major cause of acute myocardial injury characterized by a failure in regional contraction of the left ventricle, which is usually accompanied by a reduction in the ejection fraction, which is completely reversible. ${ }^{6,11}$ Both the clinical presentation and the electrocardiographic findings suggest an Acute Coronary Syndrome, and at the time of the angiographic study, a heart contracted in the ventriculogram with contractility disorders but with epicardial arteries without documented angiographically lesions is documented., $3,12,13$

With regard to the clinical picture, STT presents symptoms or signs of myocardial injury, sudden onset, usually related to a trigger. Most of the cases present in postmenopausal women and risk factors such as cigarette smoking, alcohol abuse, anxiety and dyslipidemia have been described. ${ }^{6,11,14}$

The diagnostic criteria for STTs updated by ESC are: Abnormalities in left ventricular or right segmental contractility that are frequently associated with a stressor, the abnormality in contractility usually corresponds to more than one vascular territory and corresponds to circumferential dysfunction, absence (ST segment elevation or depression, complete left bundle branch block, $\mathrm{T}$ wave inversion or QTc interval prolongation) during the acute phase (3 months), significant elevation of natriuretic peptides during the acute phase, elevation of discrete troponin with a conventional test that is unrelated to changes in echocardiogram, and recovery of ventricular function in cardiac images at follow-up 3-6 months. ${ }^{6}$

Several anatomic variants have been described in the STT, which depend on whether it is primary or secondary, the primary being understood as that syndrome where the morphology and cardiovascular damage is derived by cardiac symptoms, and secondary when it is a cardiac manifestation of some systemic disease. The classic compromise that has been described in $50-80 \%$ of patients corresponds to the regional alteration of left ventricular contractility with apical and mesial hypokinesia in addition to basal hypercontractility. The basal or inverted Takotsubo variant with circumferential basal hypokinesia and apical hypercontractility has also been described and the mean left ventricular variant with medioventricular hypokinesia and basal and apical hypercontractility. $6,7,15,16$

According to the diagnosis and treatment algorithms of the STT published in the year 2016 by the ESC, if the patient has the signs and symptoms that suggest the Takotsubo syndrome it is indicated to rule out the presence of coronary disease by means of diagnostic angiography with left ventriculography. Once the presence of 
coronary heart disease is ruled out, the study should be complemented with a cardiac imaging, be it an echocardiogram and/or a cardiac resonance to evaluate left ventricular morphology and function, anatomic variants, possible complications such as obstruction of the tract of left ventricular outflow, mitral insufficiency, right ventricular involvement, thrombus formation and cardiac rupture, and follow-up of ventricular function and reversible myocardial compromiso. ${ }^{6,8-10}$

In the present case, the patient was admitted for chest pain with typical features, associated with a positive cardiac echocardiogram with evidence of ischemic-necrotic cardiopathy with a motile disorder in both anterior, septal, lateral and postero-inferior apical segments. This presentation is part of the main features in the acute phase of STT where there is compromise of a large area of myocardium that extends beyond the territory of a single coronary artery and is usually characterized by symmetrical regional abnormalities that compromise the medial segments of the anterior, inferior and lateral walls in circumferential pattern. ${ }^{6,8,17,18}$ The Global Longitudinal Strain (SLG) can be used to evaluate regional myocardial function and in the case of STT it has been proposed that the pattern of distribution in the acute phase affects only the apical segments, similar to the image of the evil eye ". In addition, the radial strain is reduced along the left ventricular midcircumference as opposed to the acute myocardial infarction. ${ }^{17,19}$ In patients with the classic STT presentation, there is a decrease of the SLG from the base to the apex with a significant gradient indicating a more severe involvement of the apex, and these alterations in the regional strain improve in the early follow-up, on average at 34 days of the event. ${ }^{17,18,20}$

Of advanced cardiovascular imaging, both cardiac magnetic resonance and coronary angiography by tomography are playing an important role both in the diagnosis and management of patients with STT, since not only is the limitation of the acoustic window of the transthoracic echocardiogram and are noninvasive studies unlike cardiac catheterization and ventriculogram. Both images allow the evaluation of the morphology and ventricular function in addition to the characterization of the myocardium allowing to rule out differential diagnoses such as myocarditis and myocardial infarction with nonobstructive coronary arteries or MINOCA. The disadvantage of these studies is that they are not available in all institutions, so the paraclinic recommended as an initial imaging approach would be transthoracic echocardiography. ${ }^{17,21}$

\section{Conclusion}

Takotsubo syndrome is a rare condition of acute reversible heart failure in which an acute coronary syndrome should first be ruled out given the similarity of clinical presentation characteristics and findings in paraclinics such as cardiac biomarkers and initial echocardiographic images. In case of doubts regarding the possibility of secondary compromise to a non-obstructive effect of coronary arteries or other conditions such as myocarditis, studies with advanced images such as cardiac resonance or angiotomography should be complemented.

\section{Ethical responsibilities}

Protection of people and animals. The authors state that for this investigation no experiments have been performed on humans or animals.

\section{Confidentiality of the data}

The authors state that they have followed the protocols of their work center on the publication of patient data. Right to privacy and informed consent. The authors state that this article does not provide data on patients.

\section{Conflict of interests}

The authors declare that they have no conflicts of interest.

\section{References}

1. Sato H, Tateishi H, Uchida T. Takotsubo-type cardiomyopathy due to multivessel spasm. En: Kodama K, et al. editors. Clinical Aspect of Myocardial Injury: From Ischemia to Heart Failure. Tokyo: Kagakuhyouronsya Co; 1990. 56-64 p.

2. Tsuchihashi K, Ueshima K, Uchida $T$, et al. Transient left ventricular apical ballooning without coronary artery stenosis: a novel heart syndrome mimicking acute myocardial infarction. Angina Pectoris-Myocardial Infarction Investigations in Japan. $J$ Am Coll Cardiol. 2001;38(1):11-8.

3. Bybee KA, Kara T, Prasad A, et al. Systematic review: transient left ventricular apical ballooning: a syndrome that mimics ST-segment elevation myocardial infarction. Ann Intern Med. 2004;141(11):858-65.

4. Gianni M, Dentali F, Grandi AM, et al. Apical ballooning syndrome or takotsubo cardiomyopathy: a systematic review. Eur Heart J. 2006;27(13):1523-9.

5. Akashi YJ, Musha H, Kida K, et al. Reversible ventricular dysfunction takotsubo cardiomyopathy. Eur J Heart Fail. 2005;7(7):1171-6.

6. Lyon AR, Bossone E, Schneider B, et al. Current state of knowledge on Takotsubo syndrome: a Position Statement from the Taskforce on Takotsubo Syndrome of the Heart Failure Association of the European Society of Cardiology. Eur J Heart Fail. 2016;18(1):8-27.

7. Eitel I, von Knobelsdorff-Brenkenhoff F, Bernhardt P, et al. Clinical characteristics and cardiovascular magnetic resonance findings in stress (takotsubo) cardiomyopathy. JAMA. 2011;306(3):277-86.

8. Citro R, Rigo F, Ciampi Q, et al. Echocardiographic assessment of regional left ventricular wall motion abnormalities in patients with takotsubo cardiomyopathy: comparison with anterior myocardial infarction. Eur J Echocardiogr J. 2011;12(7):542-9.

9. Bossone E, Lyon A, Citro R, et al. Takotsubo cardiomyopathy: an integrated multi-imaging approach. Eur Heart $J$ Cardiovasc Imaging. 2014;15(4):366-77.

10. Meimoun P, Clerc J, Vincent C, et al. Non-invasive detection of tako-tsubo cardiomyopathy vs. acute anterior myocardial infarction by transthoracic Doppler echocardiography. Eur Heart $J$ Cardiovasc Imaging. 2013;14(5):464-70.

11. Sharkey SW. A Clinical Perspective of the Takotsubo Syndrome. Heart Fail Clin. 2016;12(4):507-20.

12. Sharkey SW, Windenburg DC, Lesser JR, et al. Natural history and expansive clinical profile of stress (tako-tsubo) cardiomyopathy. $\mathrm{J} \mathrm{Am}$ Coll Cardiol. 2010;55(4):333-41.

13. Sharkey SW, Maron BJ. Epidemiology and clinical profile of Takotsubo cardiomyopathy. Circ J. 2014;78(9):2119-28.

14. Deshmukh A, Kumar G, Pant S, et al. Prevalence of Takotsubo cardiomyopathy in the United States. Am Heart J. 2012;164(1):66-71.e1. 
15. HaghiD, AthanasiadisA, Papavassiliu T, etal. Right ventricular involvement in Takotsubo cardiomyopathy. Eur Heart J. 2006;27(20):2433-9.

16. Kurowski V, Kaiser A, von Hof K, et al. Apical and midventricular transient left ventricular dysfunction syndrome (tako-tsubo cardiomyopathy): frequency, mechanisms, and prognosis. Chest. 2007;132(3):809-16.

17. Citro R, Pontone G, Pace L, et al. Contemporary Imaging in Takotsubo Syndrome. Heart Fail Clin. 2016;12(4):559-75.

18. Okura H. Echocardiographic assessment of takotsubo cardiomyopathy: beyond apical ballooning. $J$ Echocardiogr. 2016;14(1):13-20.
19. Sosa S, Banchs J. Early recognition of apical ballooning syndrome by global longitudinal strain using speckle tracking imaging--the evil eye pattern, a case series. Echocardiography. 2015;32(7):1184-92.

20. Heggemann F, Weiss C, Hamm K, et al. Global and regional myocardial function quantification by two-dimensional strain in Takotsubo cardiomyopathy. Eur J Echocardiogr J. 2009;10(6):760-4.

21. Plácido R, Cunha Lopes B, Almeida AG, et al. The role of cardiovascular magnetic resonance in takotsubo syndrome. Journal of Cardiovascular Magnetic Resonance. 2016;18(1):68 